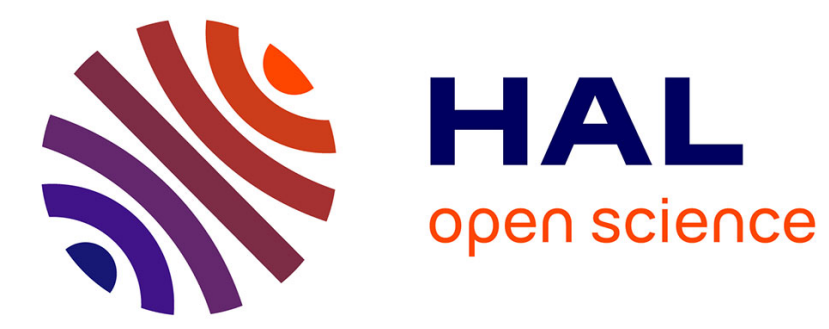

\title{
Magnetic Detector Using an Original GMR Conditioning and a Recursive Estimation of the Algebraic Derivative
}

Etienne Tisserand, Yves Berviller, Benjamin Vidales

\section{To cite this version:}

Etienne Tisserand, Yves Berviller, Benjamin Vidales. Magnetic Detector Using an Original GMR Conditioning and a Recursive Estimation of the Algebraic Derivative. IEEE Sensors Letters, 2018, 2 (4), pp.1-4. 10.1109/LSENS.2018.2875265 . hal-02079450

\section{HAL Id: hal-02079450 \\ https://hal.univ-lorraine.fr/hal-02079450}

Submitted on 3 Apr 2019

HAL is a multi-disciplinary open access archive for the deposit and dissemination of scientific research documents, whether they are published or not. The documents may come from teaching and research institutions in France or abroad, or from public or private research centers.
L'archive ouverte pluridisciplinaire HAL, est destinée au dépôt et à la diffusion de documents scientifiques de niveau recherche, publiés ou non, émanant des établissements d'enseignement et de recherche français ou étrangers, des laboratoires publics ou privés. 


\title{
Magnetic Detector Using an Original GMR Conditioning and a Recursive Estimation of the Algebraic Derivative
}

\author{
Etienne Tisserand ${ }^{1}$, Yves Berviller ${ }^{1 *}$, and Benjamin Vidales ${ }^{1}$ \\ ${ }^{1}$ Institut Jean Lamour, Université de Lorraine, Nancy, F54011, France \\ * Member, IEEE
}

\begin{abstract}
This low cost embedded system is dedicated to the detection of small variations in the magnetic flux density $B$. The measurement principle uses a giant magnetoresistance (GMR) Wheatstone bridge, whose conditioning is based on the ohmic balancing of the GMR by a virtual negative resistance connected in parallel. Associated with a capacitance, that provides a temporal base for an oscillator which delivers a frequency quasi proportional to $B$. A fast chronometry implemented on a microcontroller allows measurements in a dynamic range from 0.001 to $15 \mathrm{G}$ without a flux concentrator and in a single range. To extract useful variations, we have developed an original digital filter that estimates the algebraic derivative of the measurement signal. The convolution product between the estimating polynomial and the signal is substituted by a recursive form. This leads to a simple implementation scheme that requires only five coefficients which makes it possible to adjust the analysis time window with great flexibility. Comparative real-time tests on the magnetic detection of moving vehicles are presented.
\end{abstract}

Index Terms - Sensor Signal Processing, Digital Algebraic Derivative, GMR conditioning, Magnetic Sensor.

\section{INTRODUCTION}

Magneto-resistances made a spectacular qualitative leap with the invention of the GMR (Giant Magneto-Resistance), TMR (Tunnel Magnetic Junction) and the development of spintronics from 1990 [1-2]. Combining sensitivity, speed and very small dimensions, GMRs are now commonly used in many applications (current and position sensing, magnetometry,...) [3].

The goal of this study is to improve the sensitivity of the GMR Wheatstone bridge based detectors. Two aspects are investigated: bridge conditioning and digital processing of measurements.

In order to increase the measurement dynamics, we do not use a differential amplifier but a conditioner based on a negative impedance converter associated with an oscillator. The latter generates a signal whose frequency is proportional to the magnetic flux density $B$.

The performances of GMRs are affected by different noises (Johnson, shot and 1/f) and by drift and hysteresis phenomena that compromise accuracy and stability of measurements [4]. In detection applications, it is necessary to perform a digital filtering to extract the useful variations of the signal. An algebraic approach developed some twenty years ago leads to better performing derivation estimators that are well suited for noisy measurement signals [5-6]. This method estimates the second coefficient $\left(a_{1}\right)$ of the Taylor polynomial that fits the signal in a time window of a given width. The estimation takes the form of a running integration of the product between the signal and a particular polynomial. In order to reduce the computing load, we propose a new fully recursive estimation algorithm suitable for an implementation on embedded systems.

\section{GMR WHEATSTONE BRIDGE CONDITIONING}

\section{A. Traditional Conditioning With Differential Amplifier}

Corresponding author: Etienne Tisserand (etienne.tisserand@univ-lorraine.fr).
The GMR Wheatstone bridges used in this study are the AA family circuits manufactured by NVE Corp. The full bridge is made from 4 GMRs, two of which are magnetically shielded. In the ideal case, the characteristic $R(B)$ of a GMR is written

$$
R(B)=\left\{\begin{array}{c}
(1-\alpha(B)) R_{0} \text { for }|B|<B_{S} \\
R_{S} \text { for }|B|>B_{S}
\end{array}\right.
$$

$R_{0}$ and $R_{S}$ are respectively the resistance value at null field and the minimal value of the GMR. $B_{S}$ is the saturation density of the GMR

$$
\alpha(B)=\frac{R_{0}-R_{S}}{R_{0}} \frac{|B|}{B_{S}}
$$

For an AA002 $R_{0}=5.05 \mathrm{k} \Omega, R_{S}=4.3 \mathrm{k} \Omega$ and $B_{S}=15 G$.

The classical conditioning use a differential amplifier. If the GMRs are identical and the shielding is hermetic, we obtain

$$
\frac{U_{S}}{V_{C C}}=-\frac{A_{D}}{2} \frac{\alpha}{1-0.5 \alpha}^{\alpha<<1} \approx-\frac{A_{D}}{2} \alpha
$$

$A_{D}$ : amplification factor $\mathrm{U}_{\mathrm{S}}, V_{C C}$ : output and supply voltages

Estimation of weak fields requires a high $A_{D}$ factor which increases noise and restricts the measuring range.

\section{B. New Conditioning Approach}

We use a Negative Impedance Converter (NIC) associated with an oscillator (Fig. 1). The equivalent resistance $R_{e q}$ between the points A and GND corresponds to the parallel association of the GMR with the negative resistance synthesized by the NIC and equal to $-\frac{R_{0}}{1-\alpha}$

$$
R_{e q}=\frac{1-\alpha}{1-0.5 \alpha}{\frac{R_{0}}{2 \alpha}}^{\alpha<<1} \approx \frac{R_{0}}{2 \alpha}
$$


$R_{e q}$ is used as a resistive parameter of the Voltage Controlled Oscillator (VC0) integrated in the PLL 4046 circuit. The complete conditioner delivers a square wave with duty cycle equal to 0.5 and whose frequency $f$ depends on the magnetic field.

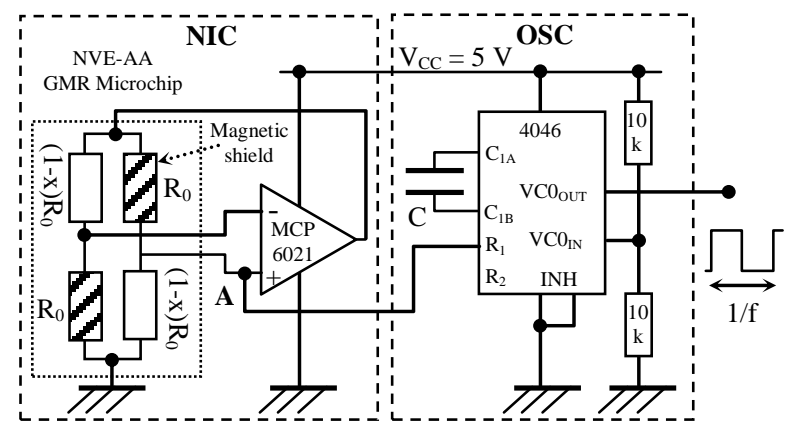

Fig. 1. GMR conditioning with NIC/OSC

With $V C O_{I N}=V_{C C} / 2, R_{I}=R_{e q}$, the oscillation frequency is written

$f(\alpha)=0.5 / R_{e q} C \approx \alpha / R_{0} C$

\section{Comparative tests}

In order to evaluate the ability of this conditioning to detect low magnetic levels, we carried out a first test which implements two AA003 bridges according to the configuration given in Fig. 2.

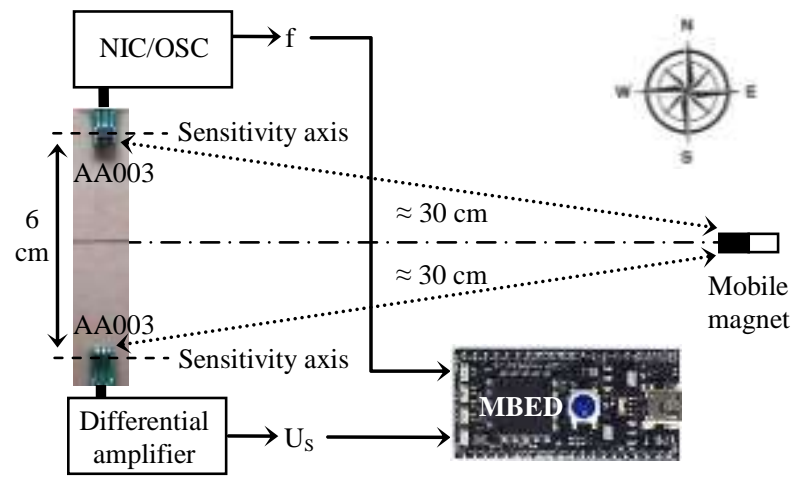

Fig. 2. Comparative test of GMR Wheatstone bridge conditioners

The first bridge is conditioned by a differential amplifier INA 126 whose gain is set at 805 . Given this high gain, the saturation of the output voltage is possible. The second bridge is conditioned by the NIC-OSC module. The capacity $\mathrm{C}$ is set at $220 \mathrm{pF}$. The oscillation frequency is about $1 \mathrm{kHz}$ under the effect of the ambient magnetic field. With the chosen value $\mathrm{C}$, the maximum VCO frequency equals $1 \mathrm{MHz}$, which guarantees an extended measuring range.

The two bridges AA003 are subjected to a magnetic variation generated by a small moving magnet. The sensors-magnet distance is $30 \mathrm{~cm}$. The contribution of this magnet according to the sensitivity axis of the sensors to the two measurement points is about $8 \mathrm{mG}$, (estimation with Vernier probe model MG-BTA, resolution $\pm 2 \mathrm{mG}$ ).

The signals $U_{S}$ and $f$ are acquired and saved by an MBED microcontroller. The frequency $f$ is determined by chronometry with a resolution of $\pm 1 \mu \mathrm{s}$. The sampling rate is set at $100 \mathrm{~Hz}$. Fig. 3 represents the variations of $U_{S}$ and $f$ when the magnet is placed and then removed from its position.
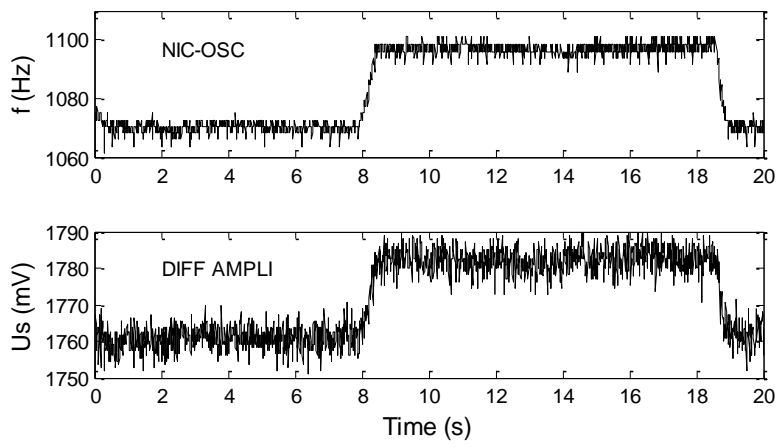

Fig. 3. Detection of a magnetic variation

The magnet generates an increase of $26 \mathrm{~Hz}$ of $f$ and $21 \mathrm{mV}$ of $U_{S}$ which corresponds to an approximate sensitivity of $3.2 \mathrm{~Hz} / \mathrm{mG}$ for the oscillator and $2.6 \mathrm{mV} / \mathrm{mG}$ for the differential amplifier.

The RMS noise level is $\pm 2 \mathrm{~Hz}$ in the first case and $\pm 5 \mathrm{mV}$ in the second case. The noise present on the frequency measurements is mainly related to the chronometric resolution.

$$
\Delta f=2 f^{2} \Delta T \approx \pm 2 \mathrm{~Hz} \text {, with } \Delta T= \pm 1 \mu \text { s and } f=1 \mathrm{kHz}
$$

In this experiment, the measurement noise floor is $0.62 \mathrm{mG}$ for the NIC-OSC against $1.9 \mathrm{mG}$ with the traditional conditioning. So, the NIC-OSC conditioning reduces by almost $10 \mathrm{~dB}$ the noise and allows a measurement of $B$ from $1 \mathrm{mG}$ to $15 \mathrm{G}$ in a single range.

The second test compares the measurement signal of our prototype to that provided by a commercial magnetometer. We use the easily available and good performing 16-bit embedded magnetometer (AMR sensor) in the LSM9DS1 inertial unit manufactured since 2015 by STMicroelectronics. Both systems are used here to detect disruptions in the earth's magnetic field generated by moving vehicles. The two magnetic detectors are placed on the sidewalk at about $2 \mathrm{~m}$ from vehicles in circulation.
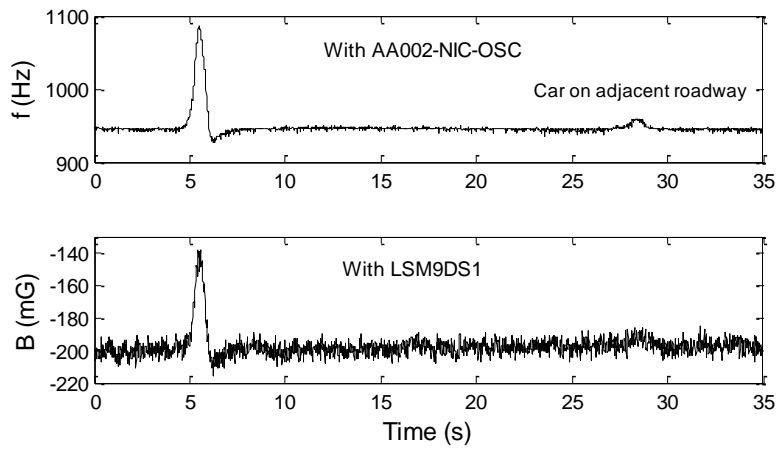

Fig. 4. Comparison of moving vehicles magnetic detection

The comparison of the signals (Fig. 4) shows the improvement of the signal-to-noise ratio provided by our system. The signal delivered by this one does not require the off-line processing used in [7] to eliminate the noise and the aberrant variations.

\section{ALGEBRAIC DERIVATIVE OF MEASURES}

\section{A. Background}

Let $x(t)$ be the measurement signal to derivate. Its Taylor series is $x(t)=a_{0}+a_{1} t / 1 !+a_{2} t^{2} / 2 !+\cdots+a_{n} t^{n} / n !+\cdots$ 
where $a_{j}=d^{j} x(0) / d t^{j}$ is the $j^{t h}$ derivate of the signal at $t=0$

For the algebraic approach this series is truncated at $n=N$, thus considering that $a_{N+1}$ is null. After some calculations in the Laplace domain, the coefficient $a_{l}$ can be isolated. Returning to the time domain and considering an observation window extending from 0 to $T$, the estimate takes the integral form

$a_{1}(t)=-\frac{24}{T^{4}} \int_{0}^{T}\left(1.5 \tau^{2}-5(T-\tau) \tau+(T-\tau)^{2}\right) \cdot x(t-\tau) d \tau$

The detailed calculations that lead to this estimation can be found in [8]. In practice, the signal is sampled and the numerical integration is provided by an FIR filter whose number of coefficients must be large enough (> 50) to ensure a good estimate.

\section{B. Recursive algebraic derivative}

Letting $\eta=\tau / T$ in (6) we obtain the normalized form

$$
a_{1}(t)=-\frac{24}{T} \int_{0}^{1}\left(7.5 \eta^{2}-7 \eta+1\right) \cdot x(t-\eta T) d \eta
$$

Expanding (7) leads to operations of the form

$$
\int_{0}^{1} x(t-\eta T) d \eta, \int_{0}^{1} \eta x(t-\eta T) d \eta \text { and } \int_{0}^{1} \eta^{2} x(t-\eta T) d \eta
$$

Integration by parts leads to the following properties

$$
\begin{aligned}
\int_{0}^{1} \eta x(t-\eta T) d \eta= & \int_{0}^{1} x(t-\eta T) d \eta-\int_{0}^{1} \int_{0}^{\eta} x\left(t-\eta^{\prime} T\right) d \eta^{\prime} d \eta=I-I I \\
\int_{0}^{1} \eta^{2} x(t-\eta T) d \eta & =\int_{0}^{1} x(t-\eta T) d \eta-2 \int_{0}^{1} \int_{0}^{\eta} x\left(t-\eta^{\prime} T\right) d \eta^{\prime} d \eta \\
& +2 \int_{0}^{1} \int_{0}^{\eta} \int_{0}^{\eta^{\prime}} x\left(t-\eta^{\prime \prime} T\right) d \eta^{\prime \prime} d \eta^{\prime} d \eta=I-2 I I+2 I I I
\end{aligned}
$$

Using these equations, the coefficients $a_{l}$ can be computed by

$$
a_{1}(t)=-\frac{24}{T}(15 \cdot I I I-8 \cdot I I+1.5 \cdot I)
$$

For $x(t)$ sampled at a frequency $f_{s}=1 / T_{s}$, let $T=M T_{s}, t=k T_{s}$, $\tau=m T_{s}, 0 \leq m \leq M-1, \eta=m / M$ and $d \eta=1 / M$

The single sliding numerical integral is defined by

$$
\hat{u}_{k}=\frac{1}{M} u_{k} \text { with } u_{k}=\sum_{m=0}^{M-1} x_{k-m}
$$

Using Z-transform, the transfer function $F(z)=U(z) / X(z)$ can be recursively written as

$$
F(z)=\sum_{m=0}^{M-1} z^{-m}=\frac{1-z^{-M}}{1-z^{-1}}
$$

The double numerical integral has a sliding intermediate upper bound. It follows relation

$$
\hat{v}_{k}=\frac{1}{M^{2}} v_{k} \text { with } v_{k}=\sum_{m=0}^{M-1} \sum_{l=0}^{m} x_{k-l}
$$

The inner sum has a transfer function of the type $F(z)$ in which $m+1$ substitutes $M$. Hence

$$
\begin{aligned}
& G(z)=\frac{V}{X}(z)=\sum_{m=0}^{M-1} \frac{1-z^{-(m+1)}}{1-z^{-1}}=\frac{1}{1-z^{-1}}\left[\sum_{m=0}^{M-1} 1-z^{-1} \sum_{m=0}^{M-1} z^{-m}\right] \\
& G(z)=\frac{V}{X}(z)=\frac{1}{1-z^{-1}}\left[M-z^{-1} \frac{1-z^{-M}}{1-z^{-1}}\right]
\end{aligned}
$$

Thus $\mathrm{G}(\mathrm{z})$ is related to $\mathrm{F}(\mathrm{z})$ by

$$
G(z)=\left(M-z^{-1} F(z)\right) /\left(1-z^{-1}\right)
$$

It should be noted that for a continuous unitary input signal $\int_{0}^{1} \int_{0}^{v} d v^{\prime} d v=\frac{1}{2}$ and $\frac{1}{M^{2}} \sum_{m=0}^{M-1} \sum_{l=0}^{m} 1=\frac{1+2+\cdots+M}{M^{2}}=\frac{1}{2}\left(1+\frac{1}{M}\right)$

The double numerical integral provides a slightly increased value. Let $D_{G}=M /(M+1)$ be the correction factor.

The triple numerical integral is expressed by

$$
\hat{w}_{k}=\frac{1}{M^{3}} w_{k} \text { with } w_{k}=\sum_{m=0}^{M-1} \sum_{n=0 l=0}^{m} \sum_{k-l}^{n} x_{k}
$$

With the same strategy, we substitute $m+1$ for $M$ in (10)

$$
\begin{aligned}
& H(z)=\frac{W}{X}(z)=\sum_{m=0}^{M-1} \frac{1}{1-z^{-1}}\left[(m+1)-z^{-1} \frac{1-z^{-(m+1)}}{1-z^{-1}}\right] \\
& H(z)=\frac{1}{1-z^{-1}}\left[\sum_{m=0}^{M-1}(m+1)-z^{-1} \sum_{m=0}^{M-1} \frac{1-z^{-(m+1)}}{1-z^{-1}}\right]
\end{aligned}
$$

Thus $H(z)$ can be determined from $G(z)$ by

$$
H(z)=\frac{M^{\prime}-z^{-1} G(z)}{1-z^{-1}} \text { with } M^{\prime}=\frac{M(M+1)}{2}
$$

For a continuous constant input, the triple numerical integral provides also an increased output with respect to the analog one.

$$
\left.\int_{0}^{1} \int_{0}^{v} \int_{0}^{v^{\prime}} d v^{\prime \prime} d v^{\prime} d v=\frac{1}{6} \text { and } \frac{1}{M^{3}} \sum_{m=0}^{M-1} \sum_{n=0 l=0}^{m} \sum_{l=\frac{1}{6}}^{n} 1=\frac{3}{M}+\frac{2}{M^{2}}\right)
$$

Let $D_{H}=M^{2} /\left(M^{2}+3 M+2\right)$ be the correction factor

Using (9), (11) and (12) in (8) provides a recursive estimate of $a_{1}$.

\section{Implementation scheme}

The nesting of the $F(z), G(z)$ et $H(z)$ transfer functions lead to the simplified implementation structure shown in Fig. 5. 


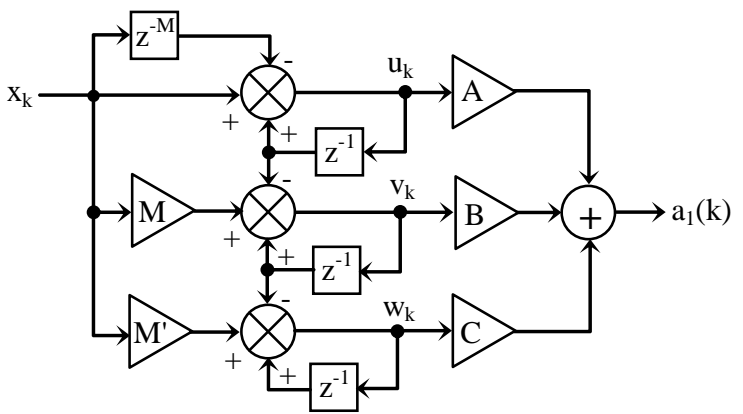

Fig. 5. Recursive structure of algebraic derivative estimator

Dividers $M, M^{2}$ and $M^{3}$ as well as correction coefficients $D_{H}$ and $D_{G}$ are put in factors $A, B$ and $C$ hence

$A=-36 f_{s} / M^{2}, B=192 f_{s} D_{G} / M^{3}$ and $C=-360 f_{s} D_{H} / M^{4}$

The number of multiplicative factors is limited to 5 compared with $M$ for a traditional FIR filter implementation,

Apart from the delay $M$, the proposed architecture does not depend on the size of the analysis window.

\section{Results of vehicle magnetic detection}

In this last test the algebraic derivative is used to detect the passage of vehicles. The filtering structure of Fig. 5 is implemented on the microcontroller. $x_{k}$ represent the frequency measurements of the oscillator (AA003-NIC-OSC). The sampling frequency equals $300 \mathrm{~Hz}$ and $M$ is set at $100\left(M^{\prime}=5050, A=-1.08, B=0.0570297\right.$ and $C=-0.0010483$ ). The coefficients $\mathrm{A}, \mathrm{B}$ and $\mathrm{C}$ are declared in double precision. The signal and its derivate $a_{l}$ delivered in real-time, are shown in Fig. 6.

These results confirm the robustness of the algebraic derivation regarding to the measurement noise. A detection of vehicles at a distance up to $3 \mathrm{~m}$ is possible without any ambiguity.

The algebraic estimator behaves as a high gain band-pass filter whose the upper cut-off frequency is inversely proportional to the analysis window width. The parameter $M$ makes it possible to select the speed range of the motions which one wishes to highlight.
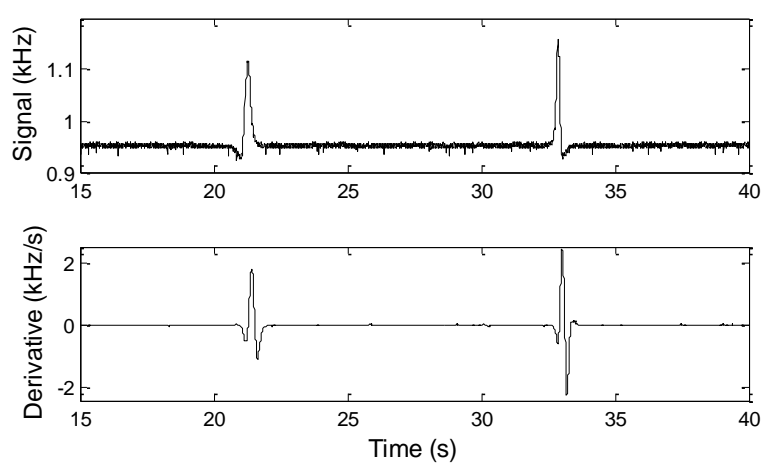

Fig. 6. Measuring signal and its first derivative

\section{REFERENCES}

[1] Freitas P, Ferreira R, Cardoso S (2016), "Spintronic sensors," Proceedings of the IEEE, vol. 104, (10), pp. 1894-1918.
[2] Bernieri A, and al. (2013), "Improving performance of GMR sensors," IEEE Sensors Journal, 2013, vol. 13, (11), pp. 4513-4520.

[3] Li, Z, Dixon S (2016), "A closed-loop operation to improve GMR sensor accuracy," IEEE Sensors Journal, vol. 16, (15), pp. 6003-6007.

[4] Baraduc C, and al. (2013), Giant Magnetoresistance Sensors, Springer, pp. 1-30.

[5] Zehetner J, Reger J, Horn M (2007) "A derivative estimation toolboxbased on algebraic methods", IEEE Intern.Conf. on Control App., 2007, pp. 331-336.

[6] Mboup M, Join C, Fliess M (2009), "Numerical differentiation with annihilators in noisy environment," Numerical Algorithms, vol. 50, (4), pp. 439-467.

[7] Yang B, Lei Y (2015), "Vehicle detection and classification for low-speed congested traffic with anisotropic magnetoresistive sensor," IEEE Sensors Journal, vol. 15, (2), pp. 1132-1138.

[8] Tisserand E, Lezama J, Schweitzer P, Berviller Y (2015), "Series arcing detection by algebraic derivative of the current," Electric Power Systems Research, vol. 119, pp. 91-99. 